\title{
Determination of Angle of Attack (AOA) for Rotating Blades
}

\author{
Shen, Wen Zhong; Hansen, Martin Otto Laver; Sørensen, Jens Nørkær
}

Published in:

Wind Energy

Publication date:

2006

Document Version

Publisher's PDF, also known as Version of record

Link back to DTU Orbit

Citation (APA):

Shen, W. Z., Hansen, M. O. L., \& Sørensen, J. N. (2006). Determination of Angle of Attack (AOA) for Rotating Blades. In Wind Energy: Proceedings of the Euromech Colloquium (pp. 205-209). Springer Verlag.

\section{General rights}

Copyright and moral rights for the publications made accessible in the public portal are retained by the authors and/or other copyright owners and it is a condition of accessing publications that users recognise and abide by the legal requirements associated with these rights.

- Users may download and print one copy of any publication from the public portal for the purpose of private study or research.

- You may not further distribute the material or use it for any profit-making activity or commercial gain

- You may freely distribute the URL identifying the publication in the public portal

If you believe that this document breaches copyright please contact us providing details, and we will remove access to the work immediately and investigate your claim 


\section{Determination of Angle of Attack (AOA) for Rotating Blades}

Wen Zhong Shen, Martin O.L. Hansen and Jens Nørkær Sørensen

\subsection{Introduction}

For a $2 \mathrm{D}$ airfoil the angle of attack (AOA) is defined as the geometrical angle between the flow direction and the chord. The concept of AOA is widely used in aero-elastic engineering models (i.e. FLEX and HAWC) as an input to tabulated airfoil data that normally are established through a combination of wind tunnel tests and corrections for the effect of Coriolis and centrifugal forces in a rotating boundary layer. For a rotating blade the flow passing by a blade section is bended due to the rotation of the rotor, and the local flow field is influenced by the bound circulation on the blade. As a further complication, $3 \mathrm{D}$ effects from tip and root vortices render a precise definition of the AOA difficult.

Today, there exists a lot of experimental and numerical (CFD) data from which precise airfoil data may be extracted. However, this leaves us with the problem of determining lift and drag polars as function of the local AOA. To determine the AOA from e.g. a computed flow field, two techniques have up to now been used. The first technique corresponds to an inverse Blade Element - Momentum (BEM) method in which a measured or computed load distribution is used as input. The technique gives reasonable results and the extracted airfoil data can be tabulated and used for later predictions using the same BEM method. Since the theory is 1D, the accuracy of the extracted airfoil data is restricted to the 1D limitation. Moreover, a BEM code needs a tip correction; different tip corrections results in different airfoil data. The second technique is the averaging technique (AT) employed in $[2,3]$. This method gives good results, especially in the middle section of a blade. Since the method utilizes averaged data, many input points are needed to evaluate the local flow features. Furthermore, it is difficult to be used for general flow conditions (e.g. at operations in yaw).

In consequence, the goal of the paper is to develop a method that can compute correctly the AOA for wind turbines in standard operations and also in general flow conditions. 


\subsection{Determination of Angle of Attack}

$\mathrm{AOA}$ is a $2 \mathrm{D}$ concept. For a rotating blade, it can be approached as an AOA in a cross-section. In order to have a uniform inflow in each cross-section, it is then selected in the azimuthal direction (i.e. at constant radius). The selected cross-section is bended in large scale but in small scale can be considered as a plane.

To determine the AOA, we need to do some preliminary work. First, the blade is divided into a few cross-sections (for example $N=10$ sections) where the blade local force is known. Second, a control point should be chosen where the velocity can be read. Theoretically, this control point can be chosen everywhere in the cross-section. In our calculation, the bound circulation around an airfoil section is considered to be a point vortex. Therefore the point should not be too close to the airfoil due to the singularity of point vortex. For convenience, it is chosen in front of the airfoil section. Since the induced velocity varies across the rotor plane, the control point should be chosen in the rotor plane. The features of the choice can be found in Fig. 37.1.

From the initial data, the AOA at each cross-section can be determined. Here we use an iterative procedure to determine the AOA:

Step 1: Determining initial flow angles using the measured velocity at every control point $\left(V_{z, i}, V_{\theta, i}\right), i \in[1, N]$

$$
\begin{gathered}
\phi_{i}^{0}=\tan ^{-1}\left(V_{z, i} / V_{\theta, i}\right), \\
V_{\mathrm{rel}, i}^{0}=\sqrt{V_{z, i}^{2}+V_{\theta, i}^{2}} .
\end{gathered}
$$

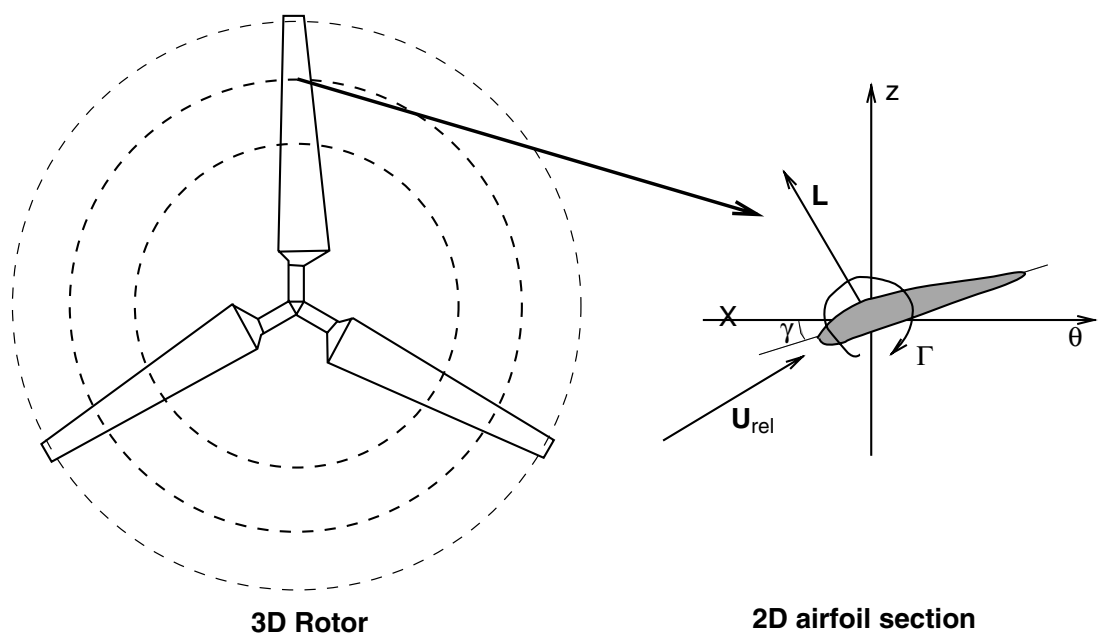

Fig. 37.1. Projection of a $3 \mathrm{D}$ rotor in $2 \mathrm{D}$ airfoil sections 
Step 2: Estimating lift and drag forces using the previous angles of attack and the measured local blade forces $\left(F_{z, i}, F_{\theta, i}\right), i \in[1, N]$

$$
\begin{aligned}
& L_{i}^{n}=F_{z, i} \cos \phi^{n}-F_{\theta, i} \sin \theta^{n}, \\
& D_{i}^{n}=F_{z, i} \sin \phi^{n}+F_{\theta, i} \cos \theta^{n} .
\end{aligned}
$$

Step 3: Computing associated circulations from the estimated lift forces as

$$
\Gamma_{i}^{n}=L_{i}^{n} /\left(\rho \sqrt{\Omega^{2} r^{2}+V_{0}^{2}}\right)
$$

where $V_{0}$ denotes wind speed and $\Omega$ denotes angular velocity of the rotor. Step 4: Computing the induced velocity created by bound vortex using circulations

$$
u_{\mathrm{in}}^{n}(x)=\left(u_{r}^{n}, u_{\theta}^{n}, u_{z}^{n}\right)=\frac{1}{4 \pi} \sum_{j=1}^{\mathrm{NB}} \int_{0}^{R} \Gamma^{n}(y) \times(x-y) /|x-y|^{3} \mathrm{~d} r .
$$

Step 5: Computing the new flow angle and the new velocity $\left(V_{z, i}-u_{z, i}^{n}\right.$, $\left.V_{\theta}-u_{\theta, i}^{n}\right)$ by subtracting the induced velocity generated by bound vortex

$$
\begin{gathered}
\phi_{i}^{n+1}=\tan ^{-1}\left(V_{z, i}-u_{z, i}^{n}\right) /\left(V_{\theta, i}-u_{\theta, i}^{n}\right), \\
V_{\mathrm{rel}, \mathrm{i}}^{n+1}=\sqrt{\left(V_{z, i}-u_{z, i}^{n}\right)^{2}+\left(V_{\theta, i}-u_{\theta, i}^{n}\right)^{2}} .
\end{gathered}
$$

Step 6: Checking the convergence criteria and deciding whether the procedure needs to go back to Step 2.

When the convergence is reached, the AOA and force coefficients can be determined:

$$
\alpha_{i}=\phi_{i}-\beta_{i}, \quad C_{d, i}=2 D_{i} / \rho V_{\mathrm{rel}, i}^{2} c_{i}, \quad C_{\mathrm{l}, i}=2 \mathrm{~L}_{i} / \rho V_{\mathrm{rel}, i}^{2} c_{i},
$$

where $\beta_{i}$ is the sum of pitch and twist angles and $c_{i}$ is the chord.

\subsection{Numerical Results and Comparisons}

In this section, the new developed method is used to determine the AOA for flows past the Tellus $95 \mathrm{~kW}$ wind turbine. The Tellus rotor is equipped with three LM8.2 blades rotating at $\omega=5.0161 \mathrm{rad} \mathrm{s}^{-1}$. Navier-Stokes computations were previous carried out for wind speeds of 5, 7, 9, 10, 12, 15, 17 and $20 \mathrm{~m} \mathrm{~s}^{-1}$ with the EllipSys3D code. For more details about CFD computations, the reader is referred to [2]. Local force on the blade and local velocity at different positions in the rotor plane are then extracted from the data. In Fig. 37.2, drag and lift coefficients at radial position $r=65.7 \% R$ are obtained 

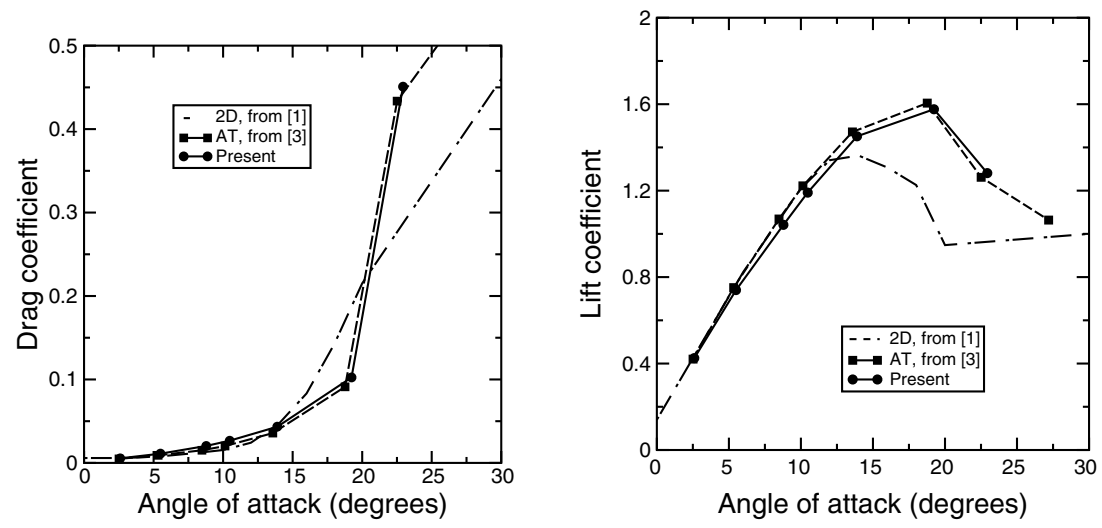

Fig. 37.2. Drag and lift coefficients at $r / R=65.7 \%$
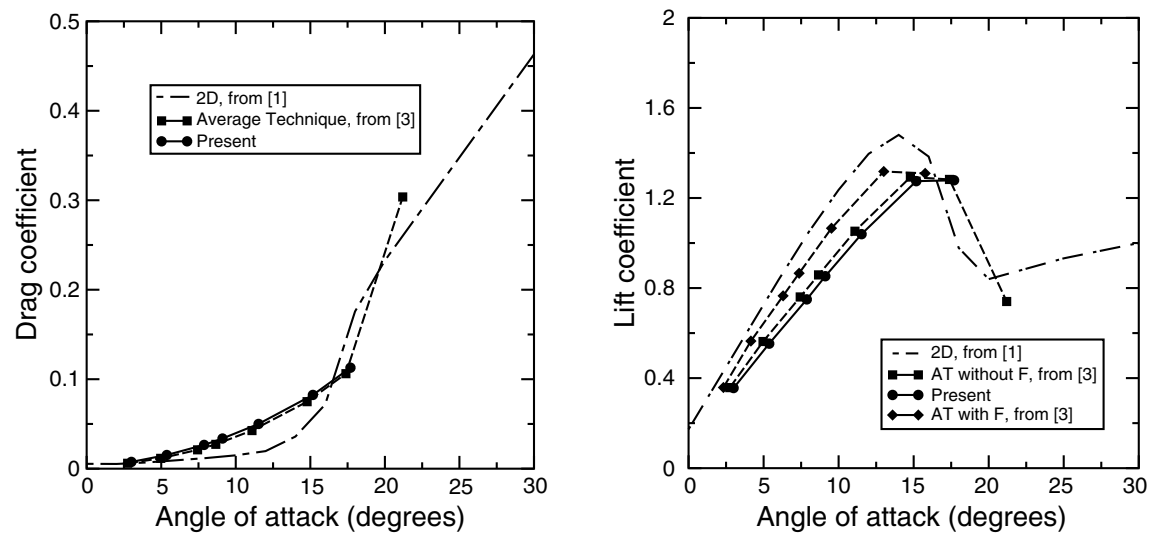

Fig. 37.3. Drag and lift coefficients at $r / R=94.7 \%$

and compared to 2D airfoil characteristics from [1] and the results of AT [2]. Excellent agreement is seen in the linear region up to $\alpha=12$. For big AOA $\left(>15^{\circ}\right), 2 \mathrm{D}$ airfoil data fails due to the Coriolis and centrifugal forces present in the boundary layer of a rotating blade. Next we consider the position closer to the blade tip at $r=94.7 \% R$. The drag and lift coefficients are plotted in Fig. 37.3. Since the position is very close to the tip, a tip correction using Prandtl's tip loss function for AT is also plotted. From the figure, we can see that the present result agrees well with the AT without tip correction. On the other hand, the introduction of a tip correction gives the curve closer to the $2 \mathrm{D}$ characteristics.

As a summary, the drag and lift coefficients in different radial positions are plotted in Fig. 37.4. From the figure, we can see that drag coefficient increases when the radial position is close to the rotor center whereas lift coefficient remains the same but with a small stall region. 

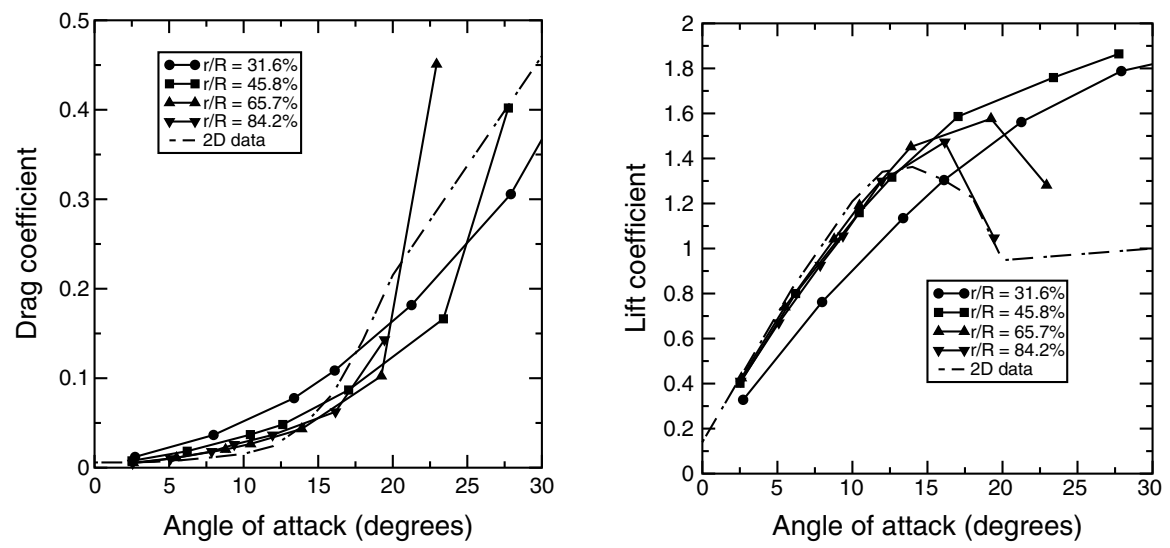

Fig. 37.4. Drag and lift coefficients in function of AOA

\subsection{Conclusion}

We have now developed a general method for determining AOA. Comparisons with the AT shows good agreements. The method can also be used to extract data from experiments (for example, with Pitot tube). Moreover it can be used to study airfoil properties in the region near the tip.

\section{References}

1. Abbott I.H., von Doenhoff A.E. (1959) Theory of wing sections. Dover, New York

2. Hansen M.O.L., Johansen J. (2004) Tip studies using CFD and computation with tip loss models. Wind Energy 7:343-356

3. Johansen J., Sørensen N.N. (2004) Airfoil characteristics from 3D CFD rotor computations. Wind Energy 7:283-294 